\title{
Non-Abelian anomalous constitutive relations of a chiral hadronic fluid *
}

\author{
Juan L. Mañes ${ }^{1, * *}$, Eugenio Megías ${ }^{2, * * *}$, Manuel Valle ${ }^{1, * * * *}$, and Miguel Á. Vázquez-Mozo ${ }^{3, \dagger}$ \\ ${ }^{1}$ Departamento de Física, Universidad del País Vasco UPV/EHU, Apartado 644, 48080 Bilbao, Spain \\ ${ }^{2}$ Departamento de Física Atómica, Molecular y Nuclear and Instituto Carlos I de Física Teórica y Com- \\ putacional, Universidad de Granada, Avenida de Fuente Nueva s/n, 18071 Granada, Spain \\ ${ }^{3}$ Departamento de Física Fundamental, Universidad de Salamanca, Plaza de la Merced s/n, 37008 Sala- \\ manca, Spain
}

\begin{abstract}
We study the constitutive relations of a chiral hadronic fluid in presence of non-Abelian 't Hooft anomalies. Analytical expressions for the covariant currents are obtained at first order in derivatives in the chiral symmetric phase, for both two and three quark flavors in the presence of chiral imbalance. We also investigate the constitutive relations after chiral symmetry breaking at the leading order.
\end{abstract}

\section{Introduction}

Quantum anomalies have important effects in relativistic hydrodynamics, as they induce extra, parity-odd terms in the constitutive relations. These anomalous contributions together with the dissipative transport effects modify the perfect fluid behavior of the constitutive relations in the form of $\left\langle J^{\mu}\right\rangle=\left\langle J^{\mu}\right\rangle_{\mathrm{PF}}+\left\langle J^{\mu}\right\rangle_{\text {diss \& anom }}$ for the charge currents, and $\left\langle T^{\mu v}\right\rangle=\left\langle T^{\mu v}\right\rangle_{\mathrm{PF}}+\left\langle T^{\mu v}\right\rangle_{\text {diss \& anom }}$ for the energy-momentum tensor [1]. Two well known examples of anomalous transport are the chiral magnetic effect (CME), consisting in the generation of an electric current driven by and parallel to an external magnetic field [2], and the chiral vortical effect (CVE) where the electric current is triggered by fluid vorticity [3], i.e. $\left\langle J^{\mu}\right\rangle_{\text {anom }}=\sigma_{\mathcal{B}} \mathcal{B}^{\mu}+\sigma_{\mathcal{V}} \omega^{\mu}$. The two associated susceptibilities, $\sigma_{\mathcal{B}}$ and $\sigma_{\mathcal{V}}$, have been computed using a wide variety of methods, for example Kubo relations [4-6], fluid/gravity correspondence [7-10], as well as the equilibrium partition function formalism (EPFF) [1113]. The latter technique is especially convenient in systems with spontaneous symmetry breaking (SSB), as in this case the constitutive relations can be obtained directly from the expression of the Bardeen-Zumino (BZ) current [14], thus avoiding the calculation of the, usually cumbersome, Wess-Zumino-Witten (WZW) functional describing the dynamics of Nambu-Goldstone (NG) bosons $[15,16]$. An example of anomalous transport phenomenon appearing only in presence of SSB is the chiral electric effect, in which the electric current is generated by an external electric field in a perpendicular direction $[17,18]$.

\footnotetext{
*Talk given by E. Megías at the vConf21: A Virtual Tribute to Quark Confinement and the Hadron Spectrum, 2-6 August 2021, online.

**e-mail: wmpmapaj@1g.ehu.es

***e-mail: emegias@ugr.es

****e-mail: manuel.valle@ehu.es

†e-mail: Miguel-Vazquez-Mozo@cern.ch
} 
Here, we apply the EPFF to study the anomalous constitutive relations in non-Abelian hadronic fluids with two [14, 18] and three [19] flavors. We will study first the fluid in the unbroken chiral symmetric phase. Later on, we will extend this study to the hydrodynamics in presence of SSB, and describe the dynamics of the NG bosons at the lowest order.

\section{Equilibrium partition function formalism}

We begin by giving a brief summary of the EPFF, which allows the evaluation of nondissipative (i.e., time-reversal odd) contributions to the constitutive relations [11, 13, 20, 21]. Let us consider the theory of a time-independent Abelian U(1) gauge connection on the stationary background

$$
\begin{aligned}
d s^{2} & \equiv G_{\mu v}(\vec{x}) d x^{\mu} d x^{v}=-e^{2 \sigma(\vec{x})}\left(d t+a_{i}(\vec{x}) d x^{i}\right)^{2}+g_{i j}(\vec{x}) d x^{i} d x^{j}, \\
\mathcal{A} & =\mathcal{A}_{0}(\vec{x}) d x^{0}+\mathcal{A}_{i}(\vec{x}) d x^{i} .
\end{aligned}
$$

The system partition function is written in terms of its Hamiltonian $H$ and the gauge charge $Q$ as $Z=\operatorname{Tr} \exp \left(-\frac{H-\mu_{0} Q}{T_{0}}\right)$, with $T_{0}$ and $\mu_{0}$ the equilibrium temperature and chemical potential. This quantity should be invariant under three-dimensional diffeomorphisms, Kaluza-Klein (KK) transformation $\left[t \rightarrow t+\phi(\vec{x}), a_{i} \rightarrow a_{i}-\partial_{i} \phi(\vec{x})\right]$, as well as $\mathrm{U}(1)$ time-independent gauge transformations, modulo possible anomalies. Moreover, KK invariance demands that $Z$ depends on the gauge fields only through the invariant combinations $A_{0} \equiv \mathcal{A}_{0}$ and $A_{i} \equiv$ $\mathcal{A}_{i}-a_{i} \mathcal{A}_{0}$. Given the general form of the partition function in terms of the background metric and field, $\log Z=\mathcal{W}\left(e^{\sigma}, A_{0}, a_{i}, A_{i}, g^{i j}, T_{0}, \mu_{0}\right)$, the expectation values of the consistent current and energy-momentum tensor can be obtained from the functional derivatives [11]

$$
\begin{array}{ll}
\left\langle J_{0}\right\rangle_{\text {cons }}=-\frac{T_{0} e^{\sigma}}{\sqrt{g_{3}}} \frac{\delta \mathcal{W}}{\delta A_{0}}, \quad\left\langle J^{i}\right\rangle_{\text {cons }}=\frac{T_{0} e^{-\sigma}}{\sqrt{g_{3}}} \frac{\delta \mathcal{W}}{\delta A_{i}}, \quad\left\langle T^{i j}\right\rangle=-\frac{2 T_{0} e^{-\sigma}}{\sqrt{g_{3}}} g^{i k} g^{j l} \frac{\delta \mathcal{W}}{\delta g^{k l}}, \\
\left\langle T_{00}\right\rangle=-\frac{T_{0} e^{\sigma}}{\sqrt{g_{3}}} \frac{\delta \mathcal{W}}{\delta \sigma}, \quad\left\langle T_{0}^{i}\right\rangle=\frac{T_{0} e^{-\sigma}}{\sqrt{g_{3}}}\left(\frac{\delta \mathcal{W}}{\delta a_{i}}-A_{0} \frac{\delta \mathcal{W}}{\delta A_{i}}\right),
\end{array}
$$

where $g_{3} \equiv \operatorname{det}\left(g_{i j}\right)$, and thus $\mathcal{W}$ plays the role of a generating functional for the constitutive relations. We study the equilibrium partition function using an expansion in derivatives, which corresponds to a hydrodynamic expansion. The general form of $\mathcal{W}$ at zeroth and first order in derivatives for the system of Eqs. (1)-(2) are respectively given by [11, 13]

$$
\begin{aligned}
& \mathcal{W}_{(0)}=\frac{1}{T_{0}} \int d^{3} x \sqrt{g_{3}} e^{\sigma} P(T, \mu), \\
& \mathcal{W}_{(1)}=\int d^{3} x \sqrt{g_{3}}\left[\alpha_{1}(T, \mu) \epsilon^{i j k} A_{i} A_{j k}+\alpha_{2}(T, \mu) \epsilon^{i j k} A_{i} f_{j k}+\alpha_{3}(T, \mu) \epsilon^{i j k} a_{i} f_{j k}\right],
\end{aligned}
$$

with $P(T, \mu)$ the pressure, and $T=e^{-\sigma} T_{0}$ and $\mu=e^{-\sigma} A_{0}$ the out-of-equilibrium temperature and chemical potential, respectively. In addition, $A_{i j} \equiv \partial_{i} A_{j}-\partial_{j} A_{i}$ and $f_{i j} \equiv \partial_{i} a_{j}-\partial_{j} a_{i}$. The zeroth order $\mathcal{W}_{(0)}$ is responsible for the perfect fluid (PF) contribution to the hydrodynamic constitutive relations, which read

$$
\left\langle J^{\mu}\right\rangle_{\mathrm{PF}}=n u^{\mu}, \quad\left\langle T^{\mu v}\right\rangle_{\mathrm{PF}}=(\varepsilon+P) u^{\mu} u^{v}+P G^{\mu v},
$$

where $\varepsilon=-P+T \frac{\partial P}{\partial T}+\mu \frac{\partial P}{\partial \mu}$ and $n=\frac{\partial P}{\partial \mu}$ are the energy density and charge density, respectively, and $u^{\mu}=e^{-\sigma}(1,0, \ldots, 0)$ is the local fluid velocity. The coefficients $\alpha_{i}(T, \mu)$ can be determined for a particular theory by inserting Eq. (6) into Eqs. (3)-(4), and comparing the 
results with the constitutive relations for this theory. The anomalous contributions to the constitutive relations at first order in derivatives turn out to be

$$
\left\langle J^{\mu}\right\rangle_{(1)}=\sigma_{\mathcal{B}} \mathcal{B}^{\mu}+\sigma_{\mathcal{V}} \omega^{\mu}, \quad\left\langle T^{\mu v}\right\rangle_{(1)}=\sigma_{\mathcal{B}}^{\varepsilon}\left(\mathcal{B}^{\mu} u^{v}+\mathcal{B}^{v} u^{\mu}\right)+\sigma_{\mathcal{V}}^{\varepsilon}\left(\omega^{\mu} u^{v}+\omega^{v} u^{\mu}\right),
$$

where the magnetic field and vorticity are $\mathcal{B}^{\mu}=\frac{1}{2} \epsilon^{\mu \nu \alpha \beta} u_{v} \mathcal{A}_{\alpha \beta}$ and $\omega^{\mu}=\frac{1}{2} \epsilon^{\mu \nu \alpha \beta} u_{v} \partial_{\alpha} u_{\beta}$, respectively. The transport coefficients in the laboratory rest frame ${ }^{1}$ for an ideal gas of Dirac fermions read $\sigma_{\mathcal{B}}=C \mu, \sigma_{\mathcal{V}}=\frac{1}{2} C \mu^{2}+C_{2} T^{2}, \sigma_{\mathcal{B}}^{\varepsilon}=\sigma_{\mathcal{V}}$ and $\sigma_{\mathcal{V}}^{\varepsilon}=\frac{1}{3} C \mu^{3}+2 C_{2} \mu T^{2}$, where $C=1 /\left(4 \pi^{2}\right)$ and $C_{2}=1 / 24$ are related to the axial anomaly $[3,8]$ and the gauge-gravitational anomaly [5, 9], respectively. Then, one finds $\alpha_{1}=-\frac{C}{6} \frac{\mu}{T}, \alpha_{2}=-\frac{1}{2}\left(\frac{C}{6} \frac{\mu^{2}}{T^{2}}-C_{2}\right)$ and $\alpha_{3}=0$. In the following we will use the EPFF extended to non-Abelian gauge fields.

\section{Non-Abelian anomalies}

We look next at a theory of chiral fermions coupled to external gauge fields $\mathcal{A}_{\mathrm{L}(\mathrm{R}) \mu} \equiv t_{a} \mathcal{A}_{\mathrm{L}(\mathrm{R}) \mu}^{a}$ with symmetry group $\mathrm{U}\left(N_{f}\right) \times \mathrm{U}\left(N_{f}\right)$, described by the Lagrangian

$$
\mathcal{L}_{\mathrm{YM}}=i \bar{\psi}_{\mathrm{L}} \gamma^{\mu}\left(\partial_{\mu}-i t_{a} \mathcal{A}_{\mathrm{L} \mu}^{a}\right) \psi_{\mathrm{L}}+i \bar{\psi}_{\mathrm{R}} \gamma^{\mu}\left(\partial_{\mu}-i t_{a} \mathcal{A}_{\mathrm{R} \mu}^{a}\right) \psi_{\mathrm{R}},
$$

with $t_{a}=t_{a}^{\dagger}$ the Lie algebra generators. The axial anomaly is signaled by the non-invariance of the effective action $\Gamma_{0}\left[\mathcal{A}_{\mathrm{L}}, \mathcal{A}_{\mathrm{R}}\right]$ under axial gauge transformations, leading to the anomaly equation $\mathscr{A}_{a}(x) \Gamma_{0}[\mathcal{V}, \mathcal{A}]=G_{a}[\mathcal{V}, \mathcal{A}]$, where $G_{a}$ is the consistent anomaly, and $\mathscr{A}_{a}(x)$ is the local generator of axial gauge transformations ${ }^{2}$. For this theory, the Bardeen form of the chiral anomaly is [23]

$$
\begin{aligned}
G_{a}[\mathcal{V}, \mathcal{A}]= & \frac{i N_{c}}{16 \pi^{2}} \epsilon^{\mu \nu \rho \sigma} \operatorname{Tr}\left\{t _ { a } \left[\mathcal{V}_{\mu \nu} \mathcal{V}_{\rho \sigma}+\frac{1}{3} \mathcal{A}_{\mu \nu} \mathcal{A}_{\rho \sigma}-\frac{32}{3} \mathcal{A}_{\mu} \mathcal{A}_{v} \mathcal{A}_{\rho} \mathcal{A}_{\sigma}\right.\right. \\
& \left.\left.+\frac{8}{3} i\left(\mathcal{A}_{\mu} \mathcal{A}_{\nu} \mathcal{V}_{\rho \sigma}+\mathcal{A}_{\mu} \mathcal{V}_{\rho \sigma} \mathcal{A}_{v}+\mathcal{V}_{\rho \sigma} \mathcal{A}_{\mu} \mathcal{A}_{v}\right)\right]\right\},
\end{aligned}
$$

where $N_{c}$ is the number of colors, while $\mathcal{V}_{\mu \nu}=\partial_{\mu} \mathcal{V}_{v}-\partial_{v} \mathcal{V}_{\mu}-i\left[\mathcal{V}_{\mu}, \mathcal{V}_{v}\right]-i\left[\mathcal{A}_{\mu}, \mathcal{A}_{v}\right]$ and $\mathcal{A}_{\mu \nu}=\partial_{\mu} \mathcal{A}_{v}-\partial_{\nu} \mathcal{A}_{\mu}-i\left[\mathcal{V}_{\mu}, \mathcal{A}_{v}\right]-i\left[\mathcal{A}_{\mu}, \mathcal{V}_{v}\right]$ are the field strengths. Notice that the nonAbelian anomaly $G_{a}$ includes contributions from the triangle, square and pentagon diagrams, in contrast to the Abelian case in which only triangle diagram contributes. The solution of the anomaly equation can be found using differential geometry methods based on the ChernSimons effective action and its dimensional reduction, with the result [14, 18, 24-26]

$$
\begin{aligned}
\Gamma_{0}[V, A]= & -\frac{N_{c}}{32 \pi^{2}} \int d t d^{3} x \sqrt{g_{3}} \epsilon^{i j k} \operatorname{Tr}\left[\frac{32}{3} i V_{0} A_{i} A_{j} A_{k}+\frac{4}{3}\left(A_{0} A_{i}+A_{i} A_{0}\right) A_{j k}\right. \\
& \left.+4\left(V_{0} A_{i}+A_{i} V_{0}\right) V_{j k}+\frac{8}{3}\left(A_{0}^{2}+3 V_{0}^{2}\right) A_{i} \partial_{j} a_{k}\right]+C_{2} T_{0}^{2} \int d t d^{3} x \sqrt{g_{3}} \epsilon^{i j k} \operatorname{Tr} A_{i} \partial_{j} a_{k},
\end{aligned}
$$

where $V_{\mu}$ and $A_{\mu}$ are KK invariant vector and axial gauge fields. From now on, we neglect the term multiplying $C_{2}$, related to the mixed gauge-gravitational anomaly.

\section{Constitutive relations in the unbroken chiral symmetric phase}

In this section we study the constitutive relations following from the effective action in Eq. (11), corresponding to the chiral symmetric phase of a QCD fluid with two and three flavors in the static geometry (1).

\footnotetext{
${ }^{1}$ This is the usual frame when computing the transport coefficients by using Kubo formulae, cf. e.g. Refs. [5, 6]. A discussion on the frame dependence of the transport coefficients can be found in e.g. Ref. [22].

${ }^{2}$ We define the vector and axial gauge fields $(\mathcal{V}, \mathcal{A})$ in terms of $\left(\mathcal{A}_{\mathrm{L}}, \mathcal{A}_{\mathrm{R}}\right)$ by $\mathcal{A}_{\mathrm{L}} \equiv \mathcal{V}-\mathcal{A}$ and $\mathcal{A}_{\mathrm{R}} \equiv \mathcal{V}+\mathcal{A}$.
} 


\subsection{Covariant currents and background}

While the consistent currents are computed from the functional derivatives of the effective action, the covariant currents are the ones entering in the constitutive relations. These latter are obtained by adding the BZ polynomials to the corresponding consistent currents, i.e. $J_{\mathrm{cov}}^{\mu}=J_{\mathrm{cons}}^{\mu}+J_{\mathrm{BZ}}^{\mu}$ [27]. Explicit expressions for $J_{\mathrm{BZ}}^{\mu}$ are provided in e.g. Refs. [14, 18, 28]. From $\mathcal{W}_{0}=i \Gamma_{0}$ and using Eqs. (3) and (4), one obtains the following equilibrium expressions [18]

$$
\begin{aligned}
\left\langle J_{a \mathrm{~V}}^{i}\right\rangle_{\mathrm{cov}} & =\frac{N_{c}}{8 \pi^{2}} e^{-\sigma} \epsilon^{i j k} \operatorname{Tr}\left\{t_{a}\left[\left(A_{0} V_{j k}+V_{j k} A_{0}\right)+\left(V_{0} A_{j k}+A_{j k} V_{0}\right)+2\left(A_{0} V_{0}+V_{0} A_{0}\right) \partial_{j} a_{k}\right]\right\}, \\
\left\langle J_{a \mathrm{~A}}^{i}\right\rangle_{\mathrm{cov}} & =\frac{N_{c}}{8 \pi^{2}} e^{-\sigma} \epsilon^{i j k} \operatorname{Tr}\left\{t_{a}\left[\left(A_{0} A_{j k}+A_{j k} A_{0}\right)+\left(V_{0} V_{j k}+V_{j k} V_{0}\right)+2\left(A_{0}^{2}+V_{0}^{2}\right) \partial_{j} a_{k}\right]\right\}, \\
\left\langle T_{0}{ }^{i}\right\rangle & =-\frac{N_{c}}{8 \pi^{2}} e^{-\sigma} \epsilon^{i j k} \operatorname{Tr}\left[\left(A_{0}^{2}+V_{0}^{2}\right) A_{j k}+\left(A_{0} V_{0}+V_{0} A_{0}\right) V_{j k}+\left(\frac{2}{3} A_{0}^{3}+2 A_{0} V_{0}^{2}\right) \partial_{j} a_{k}\right],
\end{aligned}
$$

and vanishing values for the components $\left\langle J_{a 0 \mathrm{~V}}\right\rangle_{\mathrm{cov}}=\left\langle J_{a 0 \mathrm{~A}}\right\rangle_{\mathrm{cov}}=0$ and $\left\langle T_{00}\right\rangle=\left\langle T^{i j}\right\rangle=0$. We particularize these results for a specific case of $N_{f}=2,3$. Due to the non-Abelian character of the charges, the maximal number of chemical potentials that can be introduced consistently equals the dimension of the Cartan subalgebra. Therefore, we take the background

$$
V_{\mu}(\vec{x})=V_{0 \mu}(\vec{x}) t_{0}+V_{3 \mu}(\vec{x}) t_{3}+V_{8 \mu}(\vec{x}) t_{8}, \quad A_{0}=A_{00} t_{0}, \quad A_{i}=0,
$$

where $A_{00}$ is constant, and the generators $t_{a}$ are given by

$$
\left\{\begin{array}{llll}
N_{f}=2: & t_{0}=\frac{1}{2} 1_{2 \times 2}, & t_{3}=\frac{1}{2} \sigma_{3}, & t_{8}=0_{2 \times 2}, \\
N_{f}=3: & t_{0}=\frac{1}{\sqrt{6}} 1_{3 \times 3}, & t_{3}=\frac{1}{2} \lambda_{3}, & t_{8}=\frac{1}{2} \lambda_{8}
\end{array},\right.
$$

with $\sigma_{i}$ and $\lambda_{i}$ the Pauli and Gell-Mann matrices, respectively. Furthermore, we define the equilibrium velocity field by $u_{\mu}=-e^{\sigma}\left(1, a_{i}\right)$, while the chemical potentials are $\mu_{a}=$ $e^{-\sigma} \mathcal{V}_{a 0}(a=0,3,8)$ and $\mu_{5}=e^{-\sigma} \mathcal{A}_{00}$. Here, $\mu_{5}$ controls the chiral imbalance of the system [29, 30], whereas $\mu_{8}=0$ for $N_{f}=2$. External gauge fields couple through the magnetic non-Abelian vector fields $\mathcal{B}_{a}^{\mu}=\frac{1}{2} \epsilon^{\mu \nu \alpha \beta} u_{v} \mathcal{V}_{a \alpha \beta}$. An explicit dependence on $u^{\mu}$ comes through the vorticity vector $\omega^{\mu}$.

While the constitutive relations can be expressed in the basis of the generators of the Cartan subalgebra $\left\{t_{0}, t_{3}, t_{8}\right\}$, it would be interesting to write the results also in the alternative Cartan basis formed by the conserved charges. These quantities play a fundamental role in the hydrodynamics of relativistic fluids. In the $(u d s)$ flavor sector of $\mathrm{QCD}$, the conserved charges are the baryon number $B$, electric charge $Q$, and strangeness $S$, while $S=0$ in the $(u d)$ flavor sector. In the $\{B, Q, S\}$ basis, the background defined by Eq. (15) is given by

$$
V_{\mu}(\vec{x})=V_{B \mu}(\vec{x}) B+V_{Q \mu}(\vec{x}) Q+V_{S \mu}(\vec{x}) S, \quad A_{0}=A_{B} B, \quad A_{i}=0 .
$$

Let $\Psi$ be a flavor doublet (triplet) of Dirac spinors made out of quarks, i.e.

$$
\Psi=\left(\begin{array}{l}
u \\
d
\end{array}\right) \quad \text { for } \quad N_{f}=2, \quad \text { and } \quad \Psi=\left(\begin{array}{c}
u \\
d \\
s
\end{array}\right) \text { for } \quad N_{f}=3,
$$

with each spinor being decomposed into its chiralities according to $q=\left(q_{L} q_{R}\right)^{T}$. Using that $J_{a V}^{\mu}=\bar{\Psi} \gamma^{\mu} t_{a} \Psi$, we can distinguish between two (three) vector currents for $N_{f}=2(3)$, i.e. $J_{\mathrm{em}}^{\mu}=e \bar{\Psi} \gamma^{\mu} Q \Psi, J_{B}^{\mu}=\bar{\Psi} \gamma^{\mu} B \Psi$ and $J_{S}^{\mu}=\bar{\Psi} \gamma^{\mu} S \Psi$, corresponding to the electromagnetic, baryonic and strangeness currents, respectively. These currents, together with the isospin current $J_{I}^{\mu}=\bar{\Psi} \gamma^{\mu} I_{3} \Psi$, fulfill the Gell-Mann-Nishijima relation $J_{\mathrm{em}}^{\mu}=e J_{I}^{\mu}+\frac{e}{2}\left(J_{B}^{\mu}+J_{S}^{\mu}\right)$. By using the relation between the charges $\{B, Q, S\}$ and the generators $t_{a}$ of the Cartan subalgebra of $\mathrm{U}\left(N_{f}\right)$, we will provide explicit expressions for the constitutive relations of $J_{\mathrm{em}}^{\mu}$ and $T^{\mu v}$. 


\subsection{Constitutive relations for $N_{f}=2$}

The constitutive relations can be computed by particularizing Eqs. (12)-(14) to the background of Eqs. (15)-(16) with $N_{f}=2$. While these relations are in equilibrium, the corresponding out-of-equilibrium expressions are obtained by Lorentz covariantization, so that in the end they will be expressed in terms of $\mathcal{B}_{a}^{\mu}$ and $\omega^{\mu}$. The result is

$$
\begin{aligned}
\left\langle J_{a \mathrm{~V}}^{\mu}\right\rangle_{\mathrm{cov}} & =\frac{N_{c}}{8 \pi^{2}} \mu_{5} \mathcal{B}_{0}^{\mu}, \quad(a=0,3) \\
\left\langle J_{0 \mathrm{~A}}^{\mu}\right\rangle_{\mathrm{cov}} & =\frac{N_{c}}{8 \pi^{2}}\left(\mu_{0} \mathcal{B}_{0}^{\mu}+\mu_{3} \mathcal{B}_{3}^{\mu}+\left(\mu_{0}^{2}+\mu_{3}^{2}-\mu_{5}^{2}\right) \omega^{\mu}\right), \\
\left\langle J_{3 \mathrm{~A}}^{\mu}\right\rangle_{\mathrm{cov}} & =\frac{N_{c}}{8 \pi^{2}}\left(\mu_{3} \mathcal{B}_{0}^{\mu}+\mu_{0} \mathcal{B}_{3}^{\mu}+2 \mu_{0} \mu_{3} \omega^{\mu}\right), \\
q^{\mu} & =\frac{N_{c}}{8 \pi^{2}} \mu_{5}\left[\mu_{0} \mathcal{B}_{0}^{\mu}+\mu_{3} \mathcal{B}_{3}^{\mu}+\left(\mu_{0}^{2}+\mu_{3}^{2}-\frac{1}{3} \mu_{5}^{2}\right) \omega^{\mu}\right],
\end{aligned}
$$

where $\left\langle T^{\mu v}\right\rangle=u^{\mu} q^{v}+u^{v} q^{\mu}$. The charges $\{B, Q\}$ are related to the generators $\left\{t_{0}, t_{3}\right\}$ by

$$
B=\frac{2}{3} t_{0}, \quad Q=\frac{1}{3} t_{0}+t_{3} .
$$

In the $\{B, Q\}$ basis, the background defined by Eq. (15) is given by Eq. (17) with $V_{B \mu}=$ $\frac{3}{2} V_{0 \mu}-\frac{1}{2} V_{3 \mu}, V_{Q \mu}=V_{3 \mu}, V_{S \mu}=0$ and $A_{B \mu}=\frac{3}{2} A_{0 \mu}$. Besides, the baryon and electromagnetic chemical potentials are defined by $\mu_{B}=e^{-\sigma} \mathcal{V}_{B 0}$ and $\mu_{Q}=e^{-\sigma} \mathcal{V}_{Q 0}$. Let us assume that the electromagnetic field is the only (physical) propagating gauge field. Then, we can define the physical magnetic field by $\mathcal{B}^{\mu}=\frac{1}{2} \epsilon^{\mu \nu \alpha \beta} u_{\nu} \mathscr{V}_{\alpha \beta}$ where the physical potential is $\mathscr{V}_{\mu}$ and its $\mathrm{KK}$ invariant form is $\mathbb{V}_{\mu}$, i.e. $\mathbb{V}_{0}=\mathscr{V}_{0}$ and $\mathbb{V}_{i}=\mathscr{V}_{i}-a_{i} \mathscr{V}_{0}$. One finds

$$
V_{B \mu}=0, \quad V_{Q \mu} \equiv e \mathbb{V}_{\mu} \quad \Longrightarrow \quad V_{0 \mu}=\frac{e}{3} \mathbb{V}_{\mu}, \quad V_{3 \mu}=e \mathbb{V}_{\mu}, \quad \mathcal{B}_{0}^{\mu}=\frac{e}{3} \mathcal{B}^{\mu}, \quad \mathcal{B}_{3}^{\mu}=e \mathcal{B}^{\mu}
$$

a result that implies the following relations between chemical potentials $\mu_{0}=\frac{1}{3} \mu_{3}=\frac{1}{3} \mu_{Q}$ with $\mu_{Q}=e \mathbb{V}_{0} e^{-\sigma}$. After using Eq. (24), the constitutive relations of $J_{\mathrm{em}}^{\mu}$ and $T^{\mu v}$ turn out to be

$$
\left\langle J_{\mathrm{em}}^{\mu}\right\rangle_{\mathrm{cov}}=\frac{5 e^{2} N_{c}}{36 \pi^{2}} \mu_{5} \mathcal{B}^{\mu}, \quad q^{\mu}=\frac{5 N_{c}}{36 \pi^{2}} \mu_{5}\left[e \mu_{Q} \mathcal{B}^{\mu}+\left(\mu_{Q}^{2}-\frac{3}{10} \mu_{5}^{2}\right) \omega^{\mu}\right] .
$$

$\left\langle J_{\mathrm{em}}^{\mu}\right\rangle_{\mathrm{cov}}$ gives the transport coefficient associated with the CME. The absence of a CVE in the vector current for $\mathrm{U}(2)_{\mathrm{V}} \times \mathrm{U}(2)_{\mathrm{A}}$ contrasts with the case $\mathrm{U}(1)_{\mathrm{V}} \times \mathrm{U}(1)_{\mathrm{A}}$, cf. Refs. [6, 12].

\subsection{Constitutive relations for $N_{f}=3$}

Extending the procedure presented in the previous section [19], one finds the following Lorentz covariant expressions of the constitutive relations for $N_{f}=3$

$$
\begin{aligned}
\left\langle J_{a \mathrm{~V}}^{\mu}\right\rangle_{\mathrm{cov}} & =\frac{N_{c}}{4 \sqrt{6} \pi^{2}} \mu_{5} \mathcal{B}_{a}^{\mu}, \quad(a=0,3,8) \\
\left\langle J_{0 \mathrm{~A}}^{\mu}\right\rangle_{\mathrm{cov}} & =\frac{N_{c}}{4 \sqrt{6} \pi^{2}}\left[\mu_{0} \mathcal{B}_{0}^{\mu}+\mu_{3} \mathcal{B}_{3}^{\mu}+\mu_{8} \mathcal{B}_{8}^{\mu}+\left(\mu_{0}^{2}+\mu_{3}^{2}+\mu_{8}^{2}-\mu_{5}^{2}\right) \omega^{\mu}\right] \\
\left\langle J_{3 \mathrm{~A}}^{\mu}\right\rangle_{\mathrm{cov}} & =\frac{N_{c}}{4 \sqrt{6} \pi^{2}}\left[\mu_{3} \mathcal{B}_{0}^{\mu}+\left(\mu_{0}+\frac{1}{\sqrt{2}} \mu_{8}\right) \mathcal{B}_{3}^{\mu}+\frac{1}{\sqrt{2}} \mu_{3} \mathcal{B}_{8}^{\mu}+2 \mu_{3}\left(\mu_{0}+\frac{1}{\sqrt{2}} \mu_{8}\right) \omega^{\mu}\right] \\
\left\langle J_{8 \mathrm{~A}}^{\mu}\right\rangle_{\mathrm{cov}} & =\frac{N_{c}}{4 \sqrt{6} \pi^{2}}\left[\mu_{8} \mathcal{B}_{0}^{\mu}+\frac{1}{\sqrt{2}} \mu_{3} \mathcal{B}_{3}^{\mu}+\left(\mu_{0}-\frac{1}{\sqrt{2}} \mu_{8}\right) \mathcal{B}_{8}^{\mu}+\left(2 \mu_{0} \mu_{8}+\frac{1}{\sqrt{2}}\left(\mu_{3}^{2}-\mu_{8}^{2}\right)\right) \omega^{\mu}\right] \\
q^{\mu} & =\frac{N_{c}}{4 \sqrt{6} \pi^{2}} \mu_{5}\left[\mu_{0} \mathcal{B}_{0}^{\mu}+\mu_{3} \mathcal{B}_{3}^{\mu}+\mu_{8} \mathcal{B}_{8}^{\mu}+\left(\mu_{0}^{2}+\mu_{3}^{2}+\mu_{8}^{2}-\frac{1}{3} \mu_{5}^{2}\right) \omega^{\mu}\right]
\end{aligned}
$$


The relation between the basis of conserved charges $\{B, Q, S\}$ and the basis $\left\{t_{0}, t_{3}, t_{8}\right\}$ is

$$
B=\sqrt{\frac{2}{3}} t_{0}, \quad Q=t_{3}+\frac{1}{\sqrt{3}} t_{8}, \quad S=-\sqrt{\frac{2}{3}} t_{0}+\frac{2}{\sqrt{3}} t_{8} .
$$

In the $\{B, Q, S\}$ basis, the background is given by Eq. (17) with $V_{B \mu}=\sqrt{\frac{3}{2}} V_{0 \mu}-\frac{1}{2} V_{3 \mu}+\frac{\sqrt{3}}{2} V_{8 \mu}$, $V_{Q \mu}=V_{3 \mu}, V_{S \mu}=-\frac{1}{2} V_{3 \mu}+\frac{\sqrt{3}}{2} V_{8 \mu}$ and $A_{B \mu}=\sqrt{\frac{3}{2}} A_{0 \mu}$, while $\mu_{B}$ and $\mu_{Q}$ are defined as in Sec. 4.2, and the strangeness chemical potential is $\mu_{S}=e^{-\sigma} \mathcal{V}_{S}$. Assuming, as above, that the electromagnetic field $\mathbb{V}_{\mu}$ is the only propagating gauge field, one finds

$$
\left\{\begin{array} { l } 
{ V _ { B \mu } = 0 , \quad V _ { S \mu } = 0 } \\
{ V _ { Q \mu } \equiv e \mathbb { V } _ { \mu } }
\end{array} \Longrightarrow \left\{\begin{array}{lll}
V_{0 \mu}=0, & V_{3 \mu}=e \mathbb{V}_{\mu}, & V_{8 \mu}=\frac{e}{\sqrt{3}} \mathbb{V}_{\mu} \\
\mathcal{B}_{0}^{\mu}=0, & \mathcal{B}_{3}^{\mu}=e \mathcal{B}^{\mu}, & \mathcal{B}_{8}^{\mu}=\frac{e}{\sqrt{3}} \mathcal{B}^{\mu}
\end{array},\right.\right.
$$

a result that leads to the relations $\mu_{0}=0$ and $\mu_{3}=\sqrt{3} \mu_{8}=\mu_{Q}$, with $\mu_{Q}=e \mathbb{V}_{0} e^{-\sigma}$. Finally, the constitutive relations for the electromagnetic current and energy-momentum tensor writes

$$
\left\langle J_{\mathrm{em}}^{\mu}\right\rangle_{\mathrm{cov}}=\frac{e^{2} N_{c}}{3 \sqrt{6} \pi^{2}} \mu_{5} \mathcal{B}^{\mu}, \quad q^{\mu}=\frac{N_{c}}{3 \sqrt{6} \pi^{2}} \mu_{5}\left[e \mu_{Q} \mathcal{B}^{\mu}+\left(\mu_{Q}^{2}-\frac{1}{4} \mu_{5}^{2}\right) \omega^{\mu}\right] .
$$

As in the case of $N_{f}=2$, there is no CVE in the vector current for $\mathrm{U}(3)_{\mathrm{V}} \times \mathrm{U}(3)_{\mathrm{A}}$.

\section{Lowest order constitutive relations in presence of SSB}

In this section we study the phase in which chiral symmetry is spontaneously broken. We consider the breaking pattern $U\left(N_{f}\right)_{\mathrm{L}} \times U\left(N_{f}\right)_{\mathrm{R}} \rightarrow U\left(N_{f}\right)_{\mathrm{V}}$, with the vector diagonal subgroup as the surviving symmetry. The WZW partition function, which describes the anomaly induced interactions among NG bosons as well as with the external field $\mathcal{A}$ [31-34], is given by $\Gamma^{\mathrm{WZW}}[\xi, \mathcal{A}]=\Gamma_{0}[\mathcal{A}]-\Gamma_{0}\left[\mathcal{A}_{g}\right][15,16,35,36]$, where $\Gamma_{0}$ is the anomalous functional in the symmetric phase, cf. Eq. (11). In this expression $\mathcal{A}_{g}=g^{-1} \mathcal{A} g+g^{-1} d g$ is the gauge field transformed by the generic element $g \equiv e^{i \xi_{a} t_{a}}$, with $\xi_{a}$ the NG boson fields. In our case, we make the identifications $\mathcal{A} \rightarrow\left(\mathcal{A}_{\mathrm{L}}, \mathcal{A}_{\mathrm{R}}\right)$ and $g \rightarrow(U, \mathbb{I})$, where $\mathbb{I}$ is the identity element. The matrix $U$ is then written in terms of the conventionally normalized NG boson fields associated with the broken $\mathrm{SU}\left(N_{f}\right)_{\mathrm{A}}$ symmetry as $U(\xi)=e^{i X(\xi)}$, where

$$
X(\xi) \equiv 2 \sum_{a=1}^{8} \xi_{a} t_{a}=\frac{\sqrt{2}}{f_{\pi}}\left(\begin{array}{ccc}
\frac{1}{\sqrt{2}} \pi^{0}+\frac{1}{\sqrt{6}} \eta_{8} & \pi^{+} & K^{+} \\
\pi^{-} & -\frac{1}{\sqrt{2}} \pi^{0}+\frac{1}{\sqrt{6}} \eta_{8} & K^{0} \\
K^{-} & \bar{K}^{0} & -\frac{2}{\sqrt{6}} \eta_{8}
\end{array}\right) \text { for } \quad N_{f}=3,
$$

with $f_{\pi} \approx 92 \mathrm{MeV}$ the pion decay constant. In the case $N_{f}=2, X(\xi)$ corresponds to the $2 \times 2$ submatrix of pions in Eq. (34) with $\eta_{8}=0$. The NG boson field $\xi_{0}$ is absent, as the U(1) symmetry is violated in QCD by non-perturbative effects. In the following we will focus on the action at the lowest order in derivatives, that reads

$$
\mathcal{W}_{(0)}=\frac{1}{T_{0}} \int d^{3} x \sqrt{g_{3}} e^{\sigma}\left[P\left(T,\left\{\mu_{a}\right\}\right)+\mathcal{L}\right],
$$

where $P$ is the pressure in absence of NG bosons, and the Lagrangian $\mathcal{L}$ contains all dependence on the NG bosons. The latter has the following expression in terms of KK invariant fields

$$
\mathcal{L}=\frac{f_{\pi}^{2}}{4} G^{\mu \nu} \operatorname{Tr}\left[D_{\mu} U\left(D_{v} U\right)^{\dagger}\right]=\frac{f_{\pi}^{2}}{4}\left[-e^{-2 \sigma} \operatorname{Tr}\left(\xi_{0} \xi_{0}^{\dagger}\right)+g^{i j} \operatorname{Tr}\left(\Phi_{i} \Phi_{j}^{\dagger}\right)\right],
$$


where the covariant derivative acting on the NG boson field reads

$$
\xi_{\mu} \equiv D_{\mu} U=\partial_{\mu} U-i \mathcal{V}_{3 \mu}\left[t_{3}, U\right]-i \mathcal{V}_{8 \mu}\left[t_{8}, U\right],
$$

and we have defined $\Phi_{i} \equiv \xi_{i}-a_{i} \xi_{0}=\partial_{i} U-i V_{3 i}\left[t_{3}, U\right]-i V_{8 i}\left[t_{8}, U\right]$. The terms proportional to $\left[t_{0}, U\right]$ are absent as $t_{0} \propto 1_{N_{f} \times N_{f}}$ commutes with $U$. By using Eqs. (23) and (31) one can express $\xi_{0}$ and $\Phi_{i}$ in terms of the conserved charges as $\xi_{0}=-i V_{Q 0}[Q, U]-i V_{S}[S, U]$ and $\Phi_{i}=\partial_{i} U-i V_{Q i}[Q, U]-i V_{S i}[S, U]$, whereas from Eqs. (24) and (32) we find that $\xi_{0}=-i e \mathbb{V}_{0}[Q, U]$ and $\Phi_{i}=\partial_{i} U-i e \mathbb{V}_{i}[Q, U]$. Then, the leading order Lagrangian of Eq. (36) governs the electromagnetic coupling of the charged mesons $\left(\pi^{ \pm}, K^{ \pm}\right)$. Finally, the constitutive relations at this order can be computed from the appropriate functional derivatives of the effective action in (35). The resulting expressions can be brought into a covariant form in terms of the metric $G_{\mu \nu}$ and the four velocity $u_{\mu}$. The results are

$$
\begin{aligned}
& \left\langle J_{0 \mu}\right\rangle_{\mathrm{PF}}=n_{0} u_{\mu}, \\
& \left\langle J_{a \mu}\right\rangle_{\mathrm{PF}}=n_{a} u_{\mu}-\frac{i f_{\pi}^{2}}{4} \operatorname{Tr}\left[\left[t_{a}, U\right] \partial_{\mu} U^{\dagger}+\left[t_{a}, U^{\dagger}\right]\left(\partial_{\mu} U-2 i \sum_{X=Q, S} V_{X \mu}[X, U]\right), \quad(a=3,8),\right. \\
& \left\langle T^{\mu \nu}\right\rangle_{\mathrm{PF}}=(\varepsilon+P) u^{\mu} u^{v}+P G^{\mu \nu}-\frac{f_{\pi}^{2}}{4} G^{\mu \alpha} G^{\nu \beta} \operatorname{Tr}\left[D_{\alpha} U\left(D_{\beta} U\right)^{\dagger}+D_{\beta} U\left(D_{\alpha} U\right)^{\dagger}\right],
\end{aligned}
$$

where $n_{a}=\frac{\partial P}{\partial n_{a}}(a=0,3,8)$ are the charge densities. Higher order contributions will be studied in a forthcoming publication [19].

\section{Conclusions}

We investigated non-dissipative transport induced by chiral imbalance in a hadronic fluid with $N_{f}=2,3$, both at zeroth and first order in the hydrodynamic expansion. By using the EPFF, we have characterized the effects induced by external electromagnetic fields and fluid vorticity. Particularizing to a background with two and three quark flavors and in presence of a chemical potential controlling chiral imbalance, $\mu_{5}$, we have obtained the constitutive relations for various covariant currents and the energy-momentum tensor in the unbroken chiral symmetric phase. These constitutive relations have been discussed in two different flavor bases: i) the basis of the Hermitian generators of the Lie algebra, $t_{a}$, and ii) the basis of conserved charges $\{B, Q, S\}$. We also studied, in the same background and at leading order, the partition function and constitutive relations in the presence of chiral symmetry breaking.

Finally, let us indicate that the general formalism developed in $[14,18]$ and presented here can be used in a wide variety of physical situations, ranging from the study of other sectors of the Standard Model as e.g. the electroweak sector [37, 38], to the physics of condensed matter systems like superfluids $[39,40]$ and other systems affected by triangle anomalies [41, 42].

\section{Acknowledgements}

The work of J.L.M. and M.V. has been supported by Spanish Science Ministry grant PGC2018-094626B-C21 (MCIU/AEI/FEDER, EU) and Basque Government grant IT979-16. The research of E.M. is supported by the project PID2020-114767GB-I00 financed by MCIN/AEI/10.13039/501100011033, by the FEDER/Junta de Andalucía-Consejería de Economía y Conocimiento 2014-2020 Operational Program under grant A-FQM-178-UGR18, by Junta de Andalucía under grant FQM-225, and by the Consejería de Conocimiento, Investigación y Universidad of the Junta de Andalucía and European Regional Development Fund (ERDF) under grant SOMM17/6105/UGR. The research of E.M. is also supported by the Ramón y Cajal Program of the Spanish MCIN under grant RYC-2016-20678. M.A.V.-M. acknowledges the financial support from the Spanish Science Ministry through research grant PGC2018-094626-BC22 (MCIU/AEI/FEDER, EU), as well as from Basque Government grant IT979-16. 


\section{References}

[1] D.E. Kharzeev, H.U. Yee, Phys. Rev. D84, 045025 (2011).

[2] K. Fukushima, D.E. Kharzeev, H.J. Warringa, Phys. Rev. D78, 074033 (2008).

[3] D.T. Son, P. Surowka, Phys. Rev. Lett. 103, 191601 (2009).

[4] D.E. Kharzeev, H.J. Warringa, Phys. Rev. D80, 034028 (2009).

[5] K. Landsteiner, E. Megías, F. Pena-Benitez, Phys. Rev. Lett. 107, 021601 (2011).

[6] K. Landsteiner, E. Megías, F. Pena-Benitez, Lect. Notes Phys. 871, 433 (2013).

[7] S. Bhattacharyya, V.E. Hubeny, S. Minwalla, M. Rangamani, JHEP 02, 045 (2008).

[8] J. Erdmenger, M. Haack, M. Kaminski, A. Yarom, JHEP 01, 055 (2009).

[9] K. Landsteiner, E. Megías, L. Melgar, F. Pena-Benitez, JHEP 09, 121 (2011).

[10] E. Megías, F. Pena-Benitez, JHEP 05, 115 (2013).

[11] N. Banerjee, J. Bhattacharya, S. Bhattacharyya, S. Jain, S. Minwalla, T. Sharma, JHEP 09, 046 (2012).

[12] K. Jensen, P. Kovtun, A. Ritz, JHEP 10, 186 (2013).

[13] E. Megías, M. Valle, JHEP 11, 005 (2014).

[14] J.L. Mañes, E. Megías, M. Valle, M.Á. Vázquez-Mozo, JHEP 11, 076 (2018).

[15] J. Wess, B. Zumino, Phys. Lett. 37B, 95 (1971).

[16] E. Witten, Nucl. Phys. B223, 422 (1983).

[17] Y. Neiman, Y. Oz, JHEP 09, 011 (2011).

[18] J.L. Mañes, E. Megías, M. Valle, M.Á. Vázquez-Mozo, JHEP 12, 018 (2019).

[19] E. Megías, M.Á. Vázquez-Mozo, to appear (2021).

[20] K. Jensen, R. Loganayagam, A. Yarom, JHEP 05, 134 (2014).

[21] S. Bhattacharyya, JHEP 08, 165 (2014).

[22] E. Megías, K. Landsteiner, F. Pena-Benitez, Acta Phys. Polon. Supp. 6, 45 (2013).

[23] W.A. Bardeen, Phys. Rev. 184, 1848 (1969).

[24] V.P. Nair, R. Ray, S. Roy, Phys. Rev. D86, 025012 (2012).

[25] K. Jensen, R. Loganayagam, A. Yarom, JHEP 05, 110 (2014).

[26] J.L. Mañes, M. Valle, M.Á. Vázquez-Mozo, JHEP 05, 209 (2021).

[27] W.A. Bardeen, B. Zumino, Nucl. Phys. B244, 421 (1984).

[28] J.L. Mañes, E. Megías, M. Valle, M.Á. Vázquez-Mozo (2021), 2103 . 14850.

[29] R. Gatto, M. Ruggieri, Phys. Rev. D85, 054013 (2012).

[30] X. Planells, A.A. Andrianov, V.A. Andrianov, D. Espriu, PoS QFTHEP2013, 049 (2013).

[31] R. Kaiser, Phys. Rev. D63, 076010 (2001).

[32] D.T. Son, M.A. Stephanov, Phys. Rev. D77, 014021 (2008).

[33] K. Fukushima, K. Mameda, Phys. Rev. D86, 071501 (2012).

[34] T. Brauner, S. Kadam, JHEP 03, 015 (2017).

[35] J.L. Mañes, Nucl. Phys. B250, 369 (1985).

[36] C.S. Chu, P.M. Ho, B. Zumino, Nucl. Phys. B475, 484 (1996).

[37] C. García-Recio, L.L. Salcedo, JHEP 07, 015 (2009).

[38] T. Brauner, O. Taanila, A. Tranberg, A. Vuorinen, JHEP 11, 076 (2012).

[39] S. Lin, Phys. Rev. D85, 045015 (2012).

[40] C. Hoyos, B.S. Kim, Y. Oz, JHEP 10, 127 (2014).

[41] G. Basar, D.E. Kharzeev, H.U. Yee, Phys. Rev. B89, 035142 (2014).

[42] K. Landsteiner, Phys. Rev. B89, 075124 (2014). 